\title{
Oxidation of $\Delta^{3}$-Carene
}

\author{
SVEN.GOSTABLOHM and GUNNAR WIDMARK
}

\author{
Institute of Organic Chemistry and Biochemistry, University of Stockholm, Sweden
}

\begin{abstract}
The autoxidation of highly purified $\Delta^{3}$-carene has been studied and oxidized samples have been analysed using a micro sorption method. The method has been found to be very sensitive and different types of gorptograms have been obtained.
\end{abstract}

$\mathrm{O}_{\mathrm{t}}^{\mathrm{n}}$ ne of the most well known properties of the terpene hydrocarbons is their tendency to autoxidatise. This quality of the technical turpentine is of fundamental importance when used for painting purposes, but on the other hand it can cause dermatological diseases on sensitive skin, especially that of house painters. Hellerström and Lundén ${ }^{1}$ have shown the dermatitis to be in direct relation to the degree of oxidation of Swedish turpentine and in investigations by Hellerström, Thyresson, Blohm and Widmark ${ }^{2,3}$ it was clearly demonstrated that only the products formed by the autoxidation are responsible for the skin activity.

In all work concerning allergy the chemicals used have to be purified to the highest degree, as even small amounts of impurities can give misleading results. In the case of terpenes it is difficult to obtain compounds free from isomers but we were kindly supplied, by Naval Stores Research Div., USA, with a sample of $\Delta^{3}$-carene isolated from $P$ inus ponderosa, which, after steam distillation, gave no variations upon micro sorption analysis according to Blohm 4. The choice of this terpene was favourable, as $\Delta^{3}$-carene is a representative compound of the Swedish turpentine from Pinus silvestris. - All our samples of $\Delta^{3}$-carene from Swedish sources gave great variations upon sorption analysis; compare the examples given in Fig. 1.

The oxidation of $\Delta^{3}$-carene was studied earlier by Rau ${ }^{5}$, who determined the rate of oxidation and showed it to be retarded by addition of pyrogallol and resorcinol. Owen and Simonsen ${ }^{6}$ isolated $l$-1-methyl-3-isopropenyl- $\Delta^{6}$. hexen-5-one from the oxidation products, and Fisher, Goldblatt et al. ${ }^{7}$ found $\Delta^{3}$-carene hydroperoxide to be an accelerator in the polymerization of rubber.

In this investigation $\Delta^{3}$-carene was allowed to react with oxygen at room temperature in presence of water vapour in an apparatus shown in Fig. 2. At 11 intervals, regulated by the amount of oxygen consumed, samples were taken out and examined immediately. The samples were analysed by the 


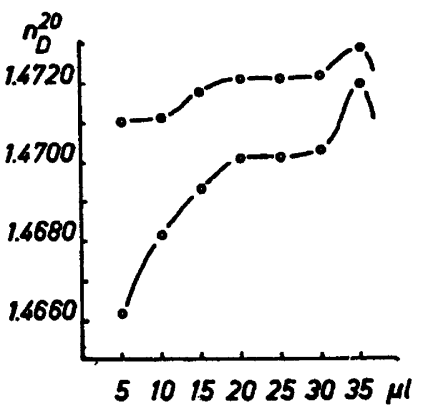

Fig. 1. Sorptograms of, commercial samples of $\Delta^{8}$-carene.

Fig. 2. Oxidation apparatus.

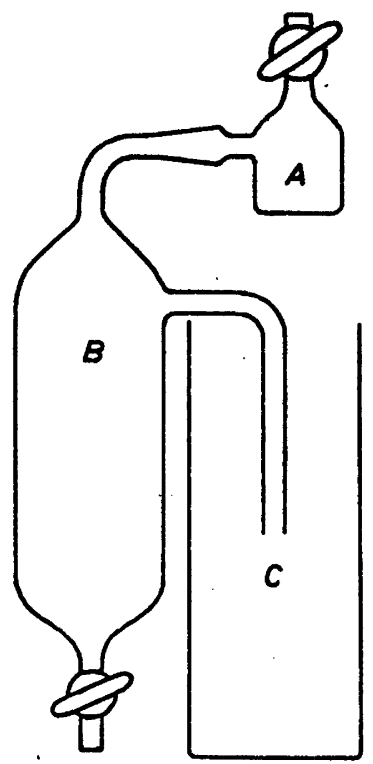

sorption method mentioned and there was found to be a marked change in the sorptograms even at the very first stage of the oxidation, thus demonstrating the sensitivity of the sorption method * (Fig. 3).

Fig. 3. Sorptograms of the oxidized sam.



* At some of the intervals mentioned samples were sealed carefully in small dark glass ampoules in an atmosphere of nitrogen and stored for two months. On sorption analysis there was a marked change over to the later stages of oxidation, thus the sorptograms of samples 6 and 9 in Fig. 3 after storing resembled those of 9 and 10. At the same time there was an increase in viscosity and a decrease in the peroxide number. - When some ampoules were opened a considerable overpressure was observed.

Acta Chem. Scand. 9 (1955) No. 6 


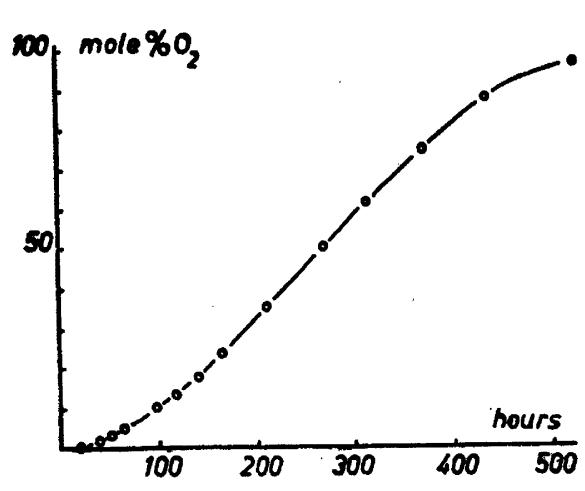

Fig. 4. Consumption of oxygen.

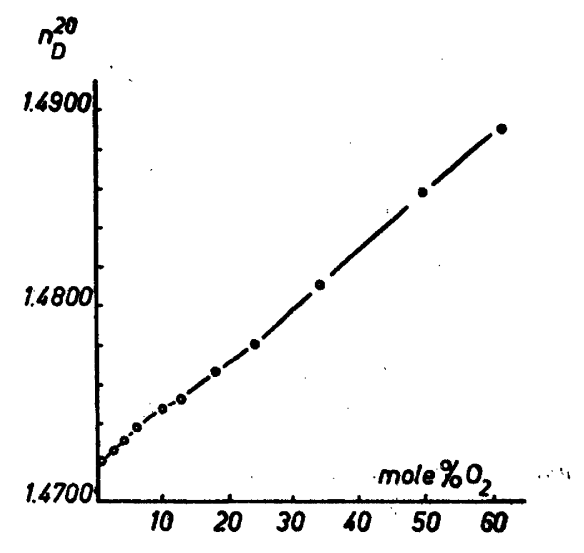

Fig. 5. Increase in refractive indices.

In connection with the sorption analysis, the viscosity and the content of the hydroperoxide were determined. As only a small amount of $\Delta^{3}$-carene was available, these determinations were performed on a micro scale. This explains the choice of methods - the flow-time of a micro pipette, and iodine titrations, respectively. We realize the disadvantages in these two methods and the results obtained, especially those concerned with the later stages of the oxidation, cannot be considered to be entirely reliable.

In the oxidation, three stages can be observed in Fig. 4: a slow phase of initiation, a middle section with a fairly steady rate of reaction, and a retarded ending. - Fig. 5 gives the increase in refractive indices. - The phase of initiation, which corresponds to a consumption of up to 5-6 mole \% oxygen samples 1 to 4 - is characterized by a slow oxidation, a very slight increase in viscosity, a marked formation of peaks at the end of the sorptograms (Fig. 3) and a direct relation (2:1) between the oxygen consumed and that liberating iodine upon titration (Fig. 6). The dermatological activity is confined to the peak fractions ${ }^{3}$. The boundary between the terpene and the displacer has a typical odour of geranium.

After this initiation, the rate of oxidation accelerates and the consumption of oxygen is almost linear against time. The relation between consumed and liberated oxygen no longer holds and less iodine is liberated in the titrations; see experimental part. There is also a marked increase in the viscosity, especially at $30-40$ mole $\%$ oxygen (cf. Fig. 7). The sorptograms give quite another figure. First the bottom of the sorptograms becomes wider and after 20 mole $\%$ of oxygen there is a sudden change with sample 6 where the first fractions have high refractive indices, and this figure characterizes the rest of the sorptograms. The samples now start to become mixed with the displacer, and even the sorption fractions taken at $50 \mu$ l give refractive indices above the value of ethanol. At 60 mole \% of oxygen the liquid becomes opalescent and is too viscous to be analysed by sorption. However, the consumption of oxygen 




Fig. 6. Relation between oxygen consumed and that liberating iodine upon titration.

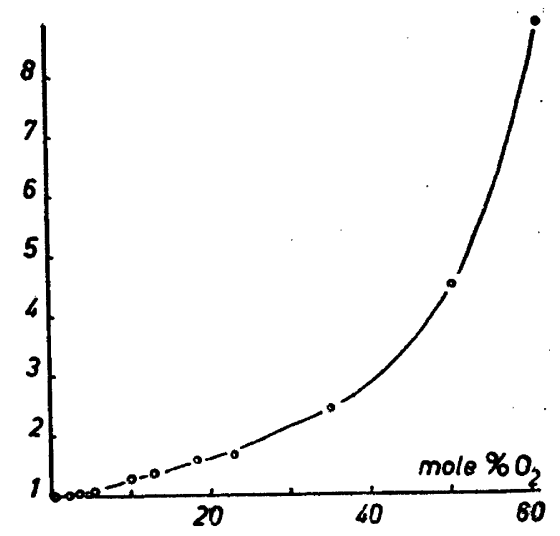

Fig. 7. Viscosity relative to pure $\Delta^{*}$. carene.

has been followed to about 100 mole \%. At 75 mole \% a yellow colour is observed and the rate of oxidation is retarded. Then, the intensity of the colour increases and the last sample investigated was a strongly yellow resin.

\section{EXPERIMENTAL PART}

Oxidation. The $\Delta^{3}$-carene used was supplied by Naval Stores Research Div., Olustee, Florida, USA, and had been isolated from ponderosa stump turpentine by vacuum distillation (100 TP, boiling point $65.5^{\circ} \mathrm{C}$ at $20 \mathrm{~mm} \mathrm{Hg}$ ). Immediately before the oxidation, the terpene was distilled with water vapour in the presence of pellets of sodium hydroxide. After separation, the liquid was dried over $\mathrm{Na}_{2} \mathrm{SO}_{4}$ and analysed. All treatments of the terpene were made in a nitrogen atmosphere.

The oxidation was carried out (in diffuse daylight) in an apparatus shown in Fig. 2. To the vessel $A$ (attached to $B$ with a long joint) was added $5 \mathrm{~g}$ of $\Delta^{3}$-carene, which had a surface of $4.15 \mathrm{~cm}^{2}$. The apparatus was filled with oxygen and the oxygen consumed by reaction with carene was replaced with water from container $C$, in such a way that the pressure remained almost constant. The temperature was controlled to $20^{\circ} \pm 2^{\circ} \mathrm{C}$.

On analysis, the samples for both the chemical and physiological tests were removed, the vessel $A$ being weighed before and after. The quantity of oxygen consumed was calculated on the amount of terpene mixture remaining. No correction was made for any water vapour which may have dissolved in the terpene.

Micro sorption analyses. The method devised by Blohm ${ }^{4}$ was followed. $40 \mu \mathrm{l}$ samples were separated into $5 \mu \mathrm{l}$ fractions by displacement sorption on silica gel. To transfer the sample quantitatively to the column, the micro pipette was rinsed twice with $40 \mu \mathrm{l}$ abs. ethanol. The column was $250 \mathrm{~mm}$ long and had an inner diameter of $1.4 \mathrm{~mm}$. Absolute ethanol was used as displacer and the gel (Davison, $2208 \times 1926$, through 200 mesh) was activated $3-5$ hours at $140^{\circ} \mathrm{C}$, at $15 \mathrm{~mm} \mathrm{Hg}$. The refractive indices of the $5 \mu \mathrm{l}$ fractions were determined according to Blohm ${ }^{8}$ (refractometer Carl Zeiss, No. 70617 , accuracy \pm 0.0001 ).

Viscosity. The micro pipette $(40 \mu \mathrm{l})$, with marks above and below the bulb, was filled with the terpene sample and suspended vertically. The temperature was $22^{\circ} \pm 1^{\circ} \mathrm{C}$. The time for the passage between the two marks was measured with a stop-watch, several drops being formed. The variations were found to be \pm 0.2 seconds and the mean value from five readings was taken. The values given in Fig. 7 have been calculated

Acta Chem. Scand. 9 (1955) No. 6 
relative to pure $\Delta^{3}$-carene, $9.0 \pm 0.2$ seconds. - With the same equipment the mean value for abs. ethenol was determined to be $9.6 \pm 0.2$ seconds.

Peroxide titrations. The determination of olefinic hydroperoxides using the iodine method has been investigated by severel authors, and many similar techniques have been given. The possible addition of iodine to the double-bond, the influence of the sample size, and the effect of oxygen on the titration are sources of error - cf. Barnard and Hargrave ${ }^{\prime}$ who have reported an accurate method, unfortunately too insensitive for our use on a micro scale. It is, however, generally accepted that the errors of the iodine titrations are reduced when performed on a micro scale - cf. Lampitt and Sylvester ${ }^{10}$.

A ground joint flask, filled with nitrogen, was weighed before and after addition of a $40 \mu \mathrm{l}$ test sample. $2.0 \mathrm{ml}$ glacial acetic acid and $1.5 \mathrm{ml}$ saturated potassium iodide solution were then added. After exactly 10 minutes, $2.0 \mathrm{ml}$ water were added and the contents were titrated with $0.002 \mathrm{~N}$ thiosulfate solution (for the last three semples $0.004-$ $0.006 \mathrm{~N}$ ), using $0.05 \mathrm{ml}$ starch solution. The thiosulfate solution was prepared immediately before use from $0.1 \mathrm{~N}$ solution. A blank was made for each titration. The procedure given is a micro modification of Yamada's ${ }^{11}$ instructions. The results are given in Fig. 6.

Acknowledgement. The investigation has been supported by the Swedish Medical Research Council.

\section{REFERENCES}

1. Hellerström, S. and Lundén, B. Acta Dermato-Venereol. 31 (1951) 1.

2. Hellerström, S., Thyreson, N., Blohm, S.- G. and Widmark, G. Acta Dermato-Venereol. 33 (1953) 51.

3. Hellerström, S., Thyreson, N., Blohm, S. G.- and Widmark, G, J. Invest. Dermatol. 24 (1955) 217.

4. Blohm, S.- G. Arkiv Kemi 6 (1954) 317.

5. Rau, M. G. Indian Forest Records 11 (1925) 197.

6. Owen, J. and Simonsen, J. L. J. Chem. Soc. 19313001.

7. Fischer, G. S., Goldblatt, L. A., Kniel, I. and Snyder, A. D. Ind. Eng. Chem. 43 (1951) 671.

8. Blohm, S.- G. Acta Chem. Scand. 4 (1950) 1495.

9. Barnard, D. and Hargrave, K. R. Anal. Chim. Acta 5 (1951) 476.

10. Lampitt, L. H. and Sylvester, N. D. Biochem. J. (London) 30 (1936) 2237.

11. Yamada, T. J. Chem. Soc. Japan, Ind. Chem. Sect. 39 (1936) 1843.

Received March 31, 1955. 